\title{
Survey on Region Growing Segmentation and Classification for Hyperspectral Images
}

\author{
S. Arokia Jerome George \\ $P G$ scholar \\ School of Computer Science, KarunyaUniversity, \\ Coimbatore, Tamilnadu.
}

\author{
S. John Livingston \\ Asst. Professor \\ School of Computer Science, Karunya University, \\ Coimbatore, Tamilnadu.
}

\begin{abstract}
Image processing of hyperspectral image sector shows a thriving upbeat in innovation of new and novel techniques. For obvious reasons, most of these apply to the process of image segmentation and classification, in which is the heart of image processing. Augmented use of hyperspectral images puts forth a hectic workload that needs to deal with spatial data imposing large memory and computing requirements. Thus, a paramount issue in image processing area is to design and implement segmentation and classification techniques demanding optimal resources. This paper presents a survey on all prominent region growing segmentation techniques analyzing each one and thus sorting out an optimal and promising technique.
\end{abstract}

\section{Keywords}

Image analysis, image segmentation, image classification.

\section{INTRODUCTION}

Image segmentation are mainly focused on the research area of image analysis. Every pixel of high spatial resolution image has different spectral signature, hyperspectral image is used for remote sensing applications including image classification. Image classification defined as a identification of objects in scene which can be captured by sensors.

Nowadays image classification have been processed each pixel independently without considering the correlations between spatially adjacent pixels[1]. In Support Vector Machine(SVM) can give good performances of classification accuracies for classifying hyperspectral images. If pixel wise classification does not take contextual information of sensed materials into account. When each pixels within a picture is created, the classification each value is not affected but our visual understanding is seriously harmed. So need to improve classification results, it is very important to develop spectralspatial classification techniques capable to consider spatial dependencies between pixels[2].

The general aim of these methods to develop for accurate classification and computational complexity of hyperspectral images. In order to achieve the accurate classification and computational complexity. It need three general strategies for hyperspectral image classification.

The first strategy develops for spectral-spatial classification using closet fixed neighborhoods. The second strategy develops for spectral spatial classification using adaptive neighborhoods. The third strategy developed for reducing over segmentations in an image.
In complicated scenes, like remotely detected pictures of the world, objects with similar spectral signatures (e.g., lakes, agricultural fields, buildings, etc.) seem in spatially separated locations. In such cases, itl's helpful to mixture these spectrally similar however spatially disjoint region objects along into teams of region objects. This modification is performed by region growing method.

\section{SEGMENTATION CLASSIFICATION HYPERSPECTRAL IMAGES}

AND

ON

There are plenty of researches has been done and plenty of method's are available in the field of segmentation for classifying hyperspectral image objects. This survey concentrates on segmentation and classification accuracy of image objects in the hyperspectral image. They are as follows.

\subsection{SVM and MRF}

This algorithm [Tarabalka et al., 2010] performs a Support Vector Machine(SVM) and Markov Random Fields(MRF) based method for accurate classification of hyperspectral images.

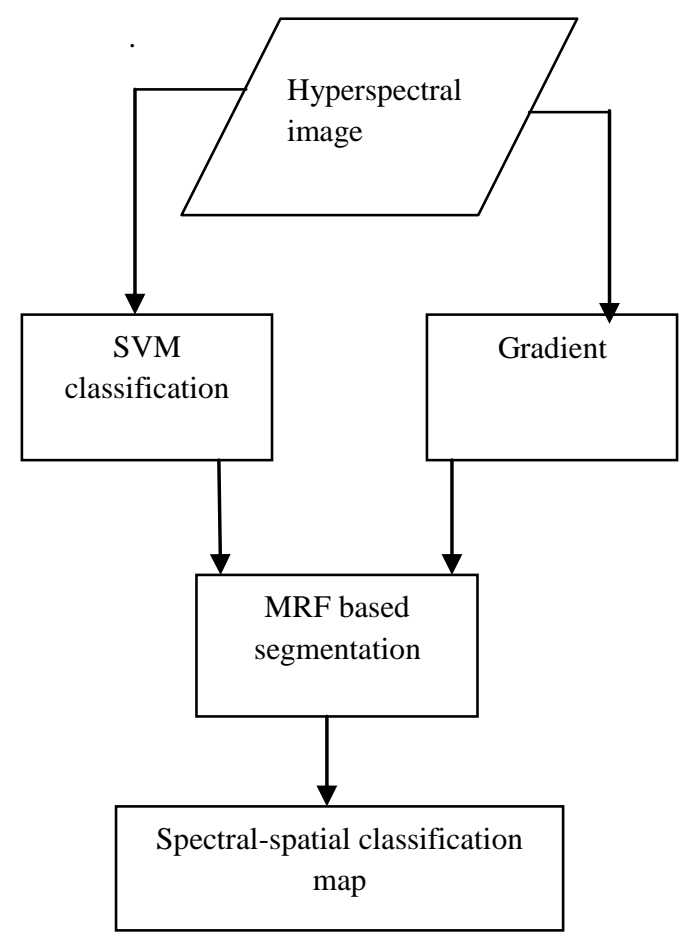

Fig1: Flowchart of SVMMRF classification 
The flowchart of SVMMRF classification is shown in Fig.1. As the input, a hyperspectral is given $X=\left\{x_{j} \in R^{B}\right.$, $j=1,2, \ldots ., n\}$. Let $\Omega=\left\{w_{1}, w_{2}, \ldots ., w_{k}\right\}$.be a set of information classes in the scene. The first step was performed a SVM pixel wise classification of the hyperspectral image [3]. SVM are mainly used for classifying hyperspectral image [4]. Next, a one band gradient of hyperspectral image is used for defining the fuzzy no edge/edge function. Here, first computed the horizontal, vertical and two diagonal gradients using sobel masks [5].The resulting gradient got from the average of four directional gradients. The final step, the SVM classification map was performed by post regularization, which means that a pixel belonging to a class is to have neighboring pixels belonging to the same class. The drawback is that preserve small structures and edges in the classification map. It is challenging task for computing accurate edge information into the classification map. For that reasons, it can be got by thresholding the gradient image

\subsection{Watershed Transformation}

The watershed algorithm extends to hyperspectral images [Tarabalka et al., 2009a]. As a hyperspectral input image $X=\left\{x_{j} \in R^{B}, j=1,2, \ldots, n\right\}$. A spectral band of every image is $\mathrm{X} \lambda, \lambda=1,2 \ldots, \mathrm{B}$. Before computing gradient, the feature extraction of original image can be performed by applying one of the transformation such as principle component Analysis(PCA)[6], Independent Component Analysis(ICA)[7] and Maximum Noise Fraction(MNF)[8]. From these transformation can get the one band or multiband image. If the gradient was in the multiband image, it can grouped into three categories. First, computation of vector gradient. Second, computation of multidimensional and finally, combination of watershed segmentation maps.

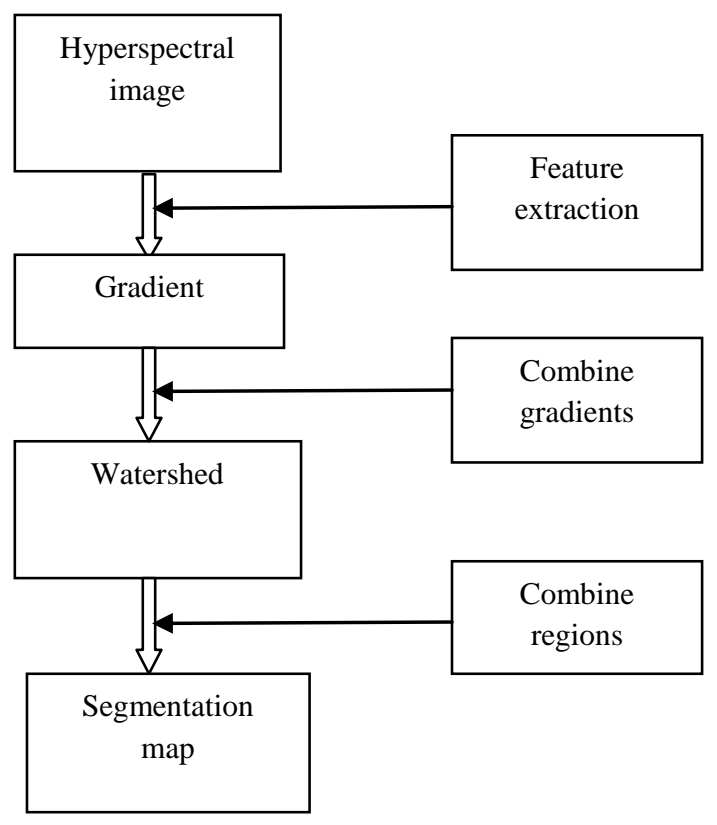

Fig2: Flowchart of watershed segmentation

Each gradient image can be performed a watershed segmentation. The watershed lines was obtained by segmentation map. In segmentation has to ensure a reliable edge detection. In segmentation map does not have information about regions but only information about edges when summing the watershed lines. In segmentation map each pixel belongs to some region without border pixel between regions. So that reason, watershed pixels assigned to one of the region in its neighborhood. A merit of this method is that led to improve classification accuracies. A demerit of this method is that small spatial structures are not identified as a separate regions.

\subsection{Partitional Clustering}

The partitional clustering algorithm extends to hyperspectral images [Tarabalka et al., 2009b]. In segmentation, the partitional clustering algorithm used for two approaches. First one is Iterative Self Organizing DATA analysis(ISODATA)[9]. Second one is Expectation Maximization(EM) to resolve the Gaussian mixture[10].



Fig3: Flowchart of partitional clustering

In partitional clustering techniques have three basic strategies. (1) In high spatial resolution image contains hundreds of spectral values, the aiming to feature extraction/selection is required for first step. In second step, clustering aims to grouping pixels, so that pixels belonging to the same clusters are spectrally similar. A similarity between vectors can be chosen by Euclidean distance[11]. The final step is grouping of pixels into a clusters. A merit of this technique is that especially suitable for classification of image with large spatial structure and improve classification accuracies. A 
demerit of this technique is that small spatial structures face a risk of being assimilated with larger neighboring structures.

\subsection{Marker-Controlled Watershed}

The marker-controlled watershed algorithm extends to hyperspectral images [Tarabalka et al., 2009c].

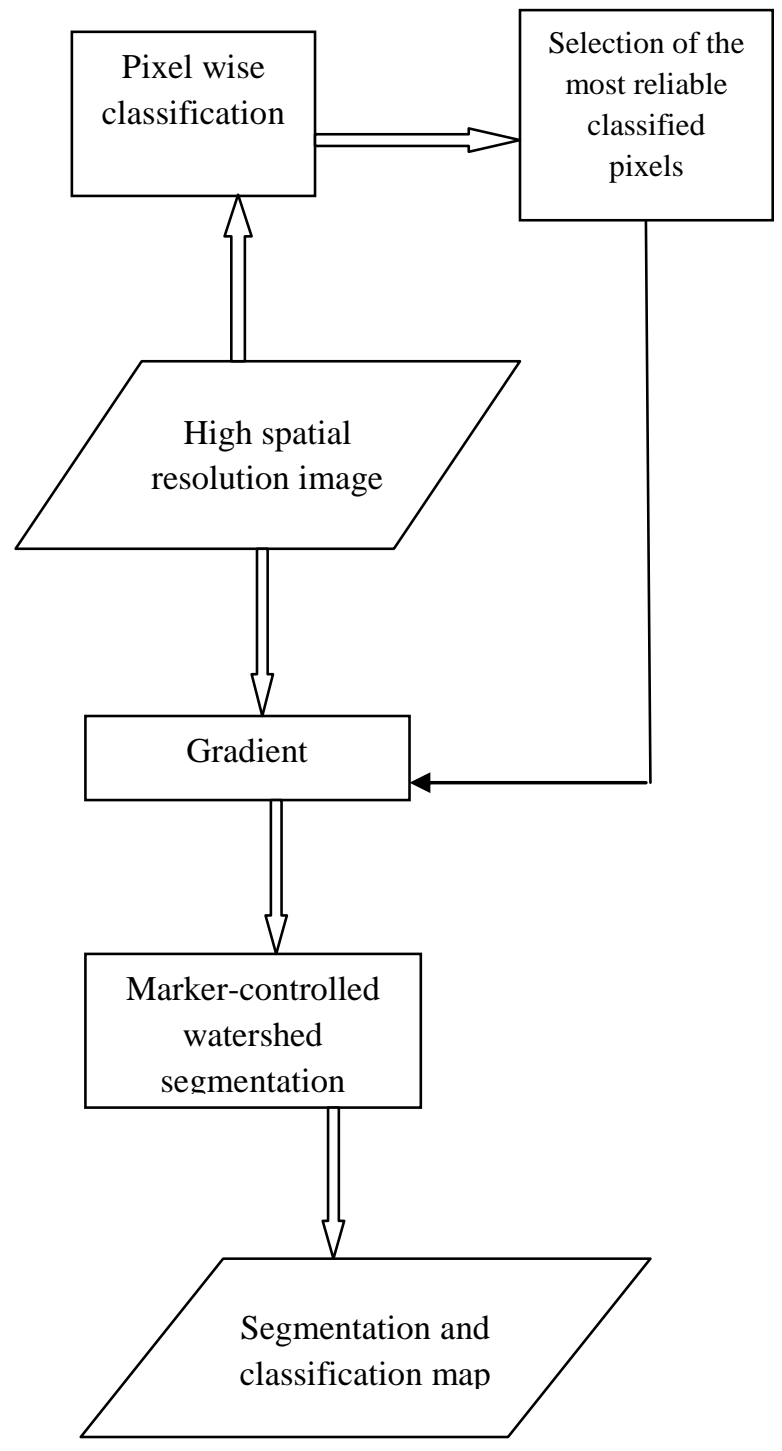

Fig4: Flowchart of marker-controlled watershed

This approach deals with hyperspectral images such that the classification and segmentation significantly reduces the computation time requirements. A hyperspectral image undergoes a pixel-wise classification map along with a probability map. Now, these probability figures are utilized to compute reliable pixels as markers. Alongside, a gradient is also computed and both are incorporated into a watershed segmentation algorithm, thus all each region pixels are allocated to respective markers producing a spectral spatial classification map.

\subsection{Automatic Marker Selection and Minimum Spanning Forest}

The construction of minimum spanning tree algorithm extends to hyperspectral images [Tarabalka et al., 2009d].



Fig5: Flowchart of automatic marker selection and minimum spanning tree

This is similar to the marker controlled segmentation approach, this algorithm works out to generate classification derived markers and do segmentation and classification processing based on the markers. An improvement is that instead of using a gradient, the image and constructing a minimum spanning forest (MSF) where markers are the roots. A polling technique is implemented to identify connected components which work together with the minimum spanning forest to generate optimal segmentation and classification map. A demerit of this method is that if no marker is chosen for a particular spatial structure, this sptial region will be lost in the final classification map.

A merit of this method is that improves classification accuracies. A demerit of this method is that if no marker is chosen for a particular spatial structure, this sptial region will be lost in the final classification map. A merit of this method is that improves classification accuracies. 


\subsection{Hierarchical Segmentation}

The hierarchical segmentation algorithm extends to hyperspectral images [Tarabalka et al., 2009e]. In Hierarchical segmentation nonadjacent region objects merging are controlled by the input parameter $S_{w t}$. This parameter values adjust from 0.0 to 1.0 .

Segmentation: Initialize the segmentation by assigning each image pixel as a reion label. Calculate the dissimilarity criterion value between all pairs of spatially adjacent regions. If threshold value is $\mathrm{T}_{\text {merge }}$ equal to the smallest dissimilarity criterion value $\mathrm{d}$ between pairs of spatially adjacent regions. Merge spatially adjacent regions with $d=T_{\text {merge. If }} S_{w t}>0$, merge pairs of non adjacent region with $d \leq S_{w t} . T_{\text {merge }}$.

Pixelwise Classification: Pixelwise classification of the segmentation map using SVM classifier.

Segmentation map: Every region in the segmentation map, all the pixels are assigned to the most frequent class with in this region using majority vote approach. A merit of Hseg is that improve classification accuracies. A demerit of Hseg is that required excessive computing time and need high computer memory requirements.

\section{Experimental Results}

The hyperspectral image[18] was taken by a experimental results. Here, classification accuracy measure based on overall accuracy, average accuracy and kappa coefficient.

Overall Accuracy(OA): It's the percentage of correctly classified pixels.

$$
\mathrm{OA}=\frac{\sum_{i}^{k} C_{i i}}{\sum_{i j}^{k} C_{i j}} X 100 \%
$$

Average Accuracy(AA): It's the mean of class specific accuracies for all the classes.

$$
\mathrm{AA}=\frac{\sum_{i}^{k} C A_{i}}{k} X 100 \%
$$

Kappa coefficient(k): It's the percentage of agreement.

$$
\begin{gathered}
\mathrm{k}=\frac{P_{o-P_{e}}}{1-P_{e}} X 100 \% \\
\mathrm{P}_{\mathrm{o}}=\mathrm{OA} / 100 \% \\
\mathrm{P}_{\mathrm{e}}=\frac{1}{N^{2}} \sum_{i}^{k} C_{i .} C_{. i} \\
\mathrm{C}_{\mathrm{i} .}=\sum_{j}^{k} C_{i j} \\
\mathrm{C}_{.}=\sum_{j}^{k} C_{j i}
\end{gathered}
$$

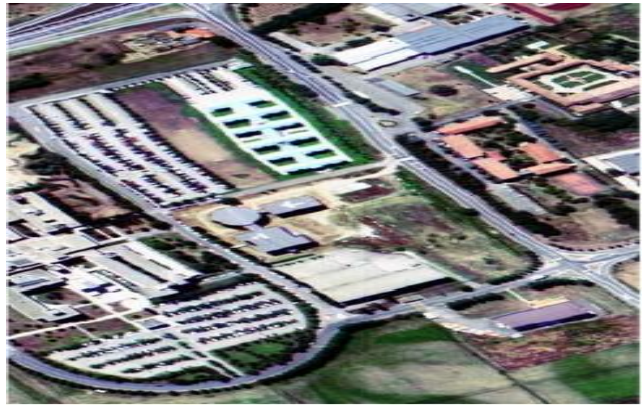

Fig6: False color hyperspectral image

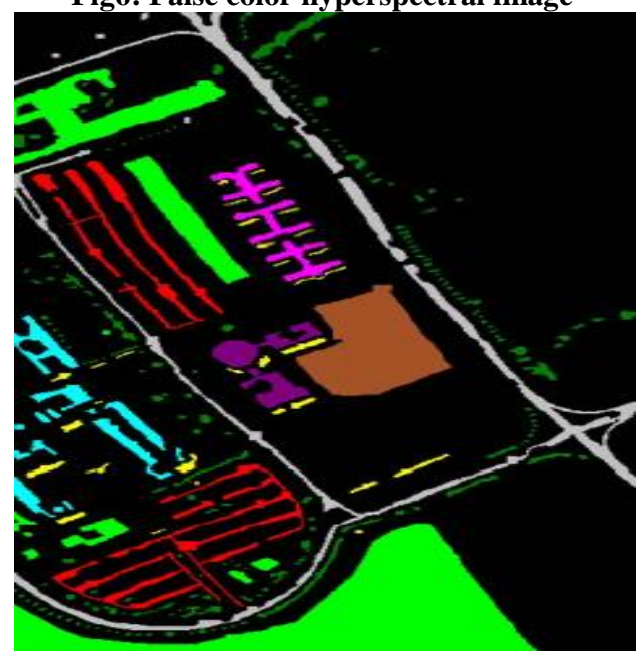

Fig7: Reference data
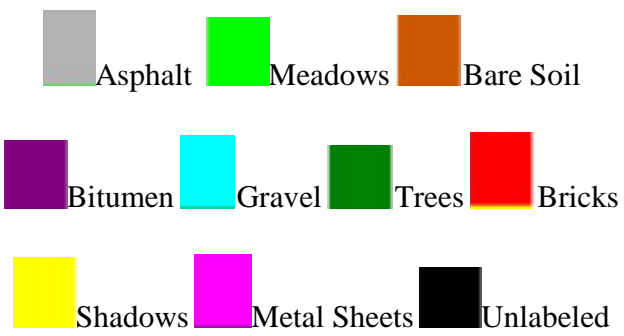

Fig8: Color key. Pixels are randomly selected from the ground reference data for SVM training and segmentation training set

pixels was randomly selected for each class from ground reference data set for SVM training data set and segmentation training set. The PV classification were found out the values of overall accuracy, Average Accuracy and Kappa Coefficient. 
Table1. Classification accuracies for SVMMRF-NE and SVMMRF-E

\begin{tabular}{|c|c|c|}
\hline & SVMMRF-NE & SVMMRF-E \\
\hline OA & 86.89 & 87.63 \\
\hline AA & 92.12 & 93.41 \\
\hline K & 83.14 & 84.07 \\
\hline Asphalt & 97.32 & 96.88 \\
\hline Meadows & 76.59 & 77.43 \\
\hline Gravel & 66.34 & 72.34 \\
\hline Trees & 99.31 & 99.28 \\
\hline Bare soil & 100 & 99.91 \\
\hline Bitumen & 95.25 & 98.12 \\
\hline Bricks & 98.57 & 97.35 \\
\hline Shadows & 97.61 & 99.46 \\
\hline Table $:$ & 99.87 \\
\hline
\end{tabular}

Table2: Classification accuracies for watershed transformation, partitional clustering and hierarchical segmentation

\begin{tabular}{|c|c|c|c|}
\hline & WH+MV & EM+MV & HSEG+MV \\
\hline OA & 85.42 & 94.00 & 93.85 \\
\hline AA & 91.31 & 93.13 & 97.07 \\
\hline K & 81.30 & 91.93 & 91.89 \\
\hline Asphalt & 93.64 & 90.10 & 94.77 \\
\hline
\end{tabular}

\begin{tabular}{|c|c|c|c|}
\hline Meadows & 75.09 & 95.99 & 89.32 \\
\hline Gravel & 66.12 & 82.26 & 96.14 \\
\hline Trees & 98.56 & 85.54 & 98.08 \\
\hline $\begin{array}{c}\text { Metal } \\
\text { sheets }\end{array}$ & 99.91 & 100 & 99.82 \\
\hline Bare soil & 97.35 & 96.72 & 99.76 \\
\hline Bitumen & 96.23 & 91.85 & 100 \\
\hline Bricks & 97.92 & 98.34 & 99.29 \\
\hline Shadows & 96.98 & 97.36 & 96.48 \\
\hline
\end{tabular}

Tabel3: Classification accuracies for marker-controlled watershed and construction of an MSF

\begin{tabular}{|c|c|c|}
\hline & WHED+MV & $\begin{array}{c}\text { SVMMSF+ } \\
\text { MV }\end{array}$ \\
\hline OA & 87.98 & 91.08 \\
\hline AA & 92.05 & 94.76 \\
\hline K & 84.32 & 88.30 \\
\hline Asphalt & 87.01 & 93.16 \\
\hline Meadows & 83.24 & 85.65 \\
\hline Gravel & 75.37 & 89.15 \\
\hline Trees & 98.97 & 91.24 \\
\hline $\begin{array}{c}\text { Metal } \\
\text { sheets }\end{array}$ & 99.91 & 99.91 \\
\hline Bare soil & 93.24 & 99.91 \\
\hline Bitumen & 95.11 & 98.57 \\
\hline Bricks & 97.00 & 99.05 \\
\hline Shadows & 98.62 & 96.23 \\
\hline
\end{tabular}

\section{COMPARISON OF THE REFERENCE PAPERS}

Table4: Compares the reference papers according to their techniques and thus identifying which approach provides the best result of segmentation and classification.

\begin{tabular}{|c|l|l|l|}
\hline $\begin{array}{c}\text { REFEREN } \\
\text { CES }\end{array}$ & \multicolumn{1}{|c|}{$\begin{array}{c}\text { METHODS } \\
\text { USED }\end{array}$} & \multicolumn{1}{|c|}{ MERITS } & \multicolumn{1}{c|}{ DEMERITS } \\
\hline$[12]$ & SVM and MRF & $\begin{array}{l}\text { Improve classification } \\
\text { accuracies }\end{array}$ & $\begin{array}{l}\text { The drawback is that preserve } \\
\text { small structures and edges in } \\
\text { the classification map. }\end{array}$ \\
\hline$[13]$ & Watershed Segmentation & $\begin{array}{l}\text { Led to improve classification } \\
\text { accuracies }\end{array}$ & $\begin{array}{l}\text { Spatial structures are not } \\
\text { identified as a separate regions. }\end{array}$ \\
\hline$[14]$ & Partitional Clustering & $\begin{array}{l}\text { Especially suitable for } \\
\text { classification of image with } \\
\text { large spatial structure and } \\
\text { improve classification } \\
\text { accuracies }\end{array}$ & $\begin{array}{l}\text { Small spatial structures face a } \\
\text { risk of being assimilated with } \\
\text { larger neighboring structures. }\end{array}$ \\
\hline$[15]$ & $\begin{array}{l}\text { Marker } \text { Controlled } \\
\text { Segmentation }\end{array}$ & $\begin{array}{l}\text { Decreases over segmentation } \\
\text { and improves classification } \\
\text { accuracies }\end{array}$ & $\begin{array}{l}\text { When creating a map of } \\
\text { markers, each marker must be } \\
\text { spatially disconnected from } \\
\text { any other marker. }\end{array}$ \\
\hline
\end{tabular}




\begin{tabular}{|c|c|c|c|}
\hline [16] & $\begin{array}{l}\text { Automatic Marker } \\
\text { Selecion And Minimum } \\
\text { Spanning Forest }\end{array}$ & $\begin{array}{l}\text { Improves } \quad \text { classification } \\
\text { accuracies }\end{array}$ & $\begin{array}{l}\text { If no marker is chosen for a } \\
\text { particular spatial structure, this } \\
\text { spatial region will be lost in the } \\
\text { final classification map }\end{array}$ \\
\hline $\begin{array}{l}{[17]} \\
\end{array}$ & $\begin{array}{l}\text { Hierarchical } \\
\text { Segmentation }\end{array}$ & $\begin{array}{ll}\text { Improve } & \text { classification } \\
\text { accuracies } & \end{array}$ & $\begin{array}{l}\text { Required excessive computing } \\
\text { time and need high computer } \\
\text { memory requirements. }\end{array}$ \\
\hline
\end{tabular}

\section{CONCLUSION}

This work was outset from the need to study and analyze various region growing image segmentation techniques on hand. A keen analysis is done on each approach to juice out their features and overheads. A major finding is that most of the techniques impose a profound level of complexity. Thus, a prominent work can be one which integrates parallel processing paradigm into the available image processing techniques to enhance the overall accuracy of the work practice.

\section{REFERENCES}

[1] Landgrebe D.A. Signal Theory Methods in Multispectral Remote Sensing. John Wiley \& Sons, Inc., 2003.

[2] Kettig R. L. and Landgrebe D. A. Classification of multispectral image data by extraction and classification of homogeneous objects. IEEE Trans. Geoscience Electronics, 14(1):19-26, Jan. 1976.

[3] Benediktsson J. A. and Swain P. H. Statistical Methods and Neural Network Approaches for Classification of Data from Multiple Sources. PhD thesis, Purdue Univ., School of Elect. Eng., West Lafayette, IN, 1990.

[4] Akçay H. G. and Aksoy S. Automatic detection of geospatial objects using multiple hierarchical segmentations. IEEE Trans. Geos. and Remote Sens., 46(7):2097-2111, July 2008.

[5] Bovolo F. and Bruzzone L. A context-sensitive technique based on support vector machines forimage classification. In Proc of. PReMI, pages 260-265, 2005.

[6] Richards J. A. and Jia X. Remote Sensing Digital Image Analysis: An Introduction. Springer- Verlag Berlin Heidelberg, 2006.

[7] Moussaoui S, Hauksdottir H, Schmidt F, Jutten C, Chanussot C, Brie D, Douté S, and Benediktsson J. A. On the decomposition of Mars hyperspectral data by ICA and Bayesian positive source separation. Neurocomputing, 71:2194-2208, 2008.

[8] Green A. A, Berman M, Switzer P, and Craig M. D. A transformation for ordering multispectral data in terms of image quality with implications for noise removal. IEEE
Trans. Geos. and Remote Sens., 26(1):65-74, Jan 1988.

[9] Ball G and Hall D. ISODATA, a novel method of data analysis and classification. Technical report, Technical Report AD-699616, Stanford University, Stanford, CA, 1965.

[10] Dempster A. P, Laird N. M, and Rubin D. B. Maximum likelihood from incomplete data via the EM algorithm. Journal of the Royal Statistical Society, B 39(1):1-38, 1977.

[11] Diday E and Simon J. C. Clustering analysis. In Digital Pattern Recognition, pages 47-94, 1976.

[12] Tarabalka Y, Fauvel M, Chanussot J, Benediktsson J. A, "SVM and MRF-based method for accurate classification of hyperspectral images,"IEEE Geoscience and Remote $\begin{array}{lll}\text { Sensing Letters, 2010, DOI } & \end{array}$ 10.1109/LGRS.2010.2047711.

[13] Tarabalka Y. and Chanussot J. and Benediktsson J. A.: "Classification based marker selection for watershed transform of hyperspectral images," in Proc. of IGARSS'09, Cape Town, South Africa, 2009.

[14] Tarabalka Y. and Benediktsson J. A. and Chanussot J.: "Spectral-spatial classification of hyperspectral imagery based on partitional clustering techniques," IEEE Trans. Geos. and Remote Sens., vol. 47, no. 8, pp. 2973-2987, Aug. 2009.

[15] Tarabalka Y. and Chanussot J. and Benediktsson J. A.: "Classification based marker selection for watershed transform of hyperspectral images," in Proc. of IGARSS'09, Cape Town, South Africa,2009,

[16] Tarabalka Y. and Chanussot J. and Benediktsson J. A.: "Classification of hyperspectral images using automatic marker selection and Minimum Spanning Forest," in Proc. of IEEE WHISPERS'09, Grenoble, France, 2009.

[17] Tarabalka Y. and Benediktsson J. A. and Chanussot J.: "Classification of hyperspectral data using Support Vector Machines and adaptive neighborhoods," in Proc. of the 6th EARSeL SIG IS workshop, Tel Aviv, Israel, 2009.

[18] Landgrebe D. A, Signal Theory Methods in Multispectral Remote Sensing. Hoboken, NJ: Wiley, 2003. 